\title{
Quaderni
}

QUADERNI Communication, technologies, pouvoir

89 | Hiver 2015-2016

Penser l'avenir : le CESTA, un think tank atypique

\section{Penser l'avenir. Un think tank atypique : le CESTA (1982-1986)}

\section{Marc Chopplet}

\section{Q OpenEdition \\ 1 Journals}

\section{Édition électronique}

URL : http://journals.openedition.org/quaderni/950

DOI : 10.4000/quaderni.950

ISSN : 2105-2956

Éditeur

Les éditions de la Maison des sciences de l'Homme

\section{Édition imprimée}

Date de publication : 5 janvier 2016

Pagination : 5-9

\section{Référence électronique}

Marc Chopplet, «Penser l'avenir. Un think tank atypique : le CESTA (1982-1986)», Quaderni [En ligne], 89 | Hiver 2015-2016, mis en ligne le 05 janvier 2016, consulté le 25 septembre 2020. URL : http:// journals.openedition.org/quaderni/950; DOI : https://doi.org/10.4000/quaderni.950 


\section{$D$ ossier}

\section{Penser l'avenir.}

«L'image authentique peut être ancienne, la pensée authentique est neuve.

Un think tank

Elle est d'aujourd'hui. Cet aujourd'hui peut être indigent, c'est vrai. Mais quel qu'il soit, il faut le prendre fermement par les cornes pour pouvoir atypique : interroger le passé ».

Walter Benjamin, « Contre un chef d'œuvre » in le CESTA Euvres II. Gallimard Folio/Essais, 2013.

Les « Futures studies » rencontrent un grand (1982-1986) succès dans les pays anglo-saxons. La World Futures Studies Federation (WFSF) créée en France par l'Unesco en 1973 est aujourd'hui active depuis l'Australie. Le Centre for Futures Studies en Grande-Bretagne se présente comme un think tank destiné à donner à ses clients publics et privés les moyens d'anticiper, de créer et de gérer leur futur tandis que l'Institut for Futures Studies en Suède est une fondation indépendante de recherche en sciences sociales travaillant de manière étroite avec le gouvernement sur des grands sujets de société. Le n ${ }^{\circ} 70$ des Quaderni consacré aux think tanks ${ }^{1}$ soulignait à la fois cette origine anglo-saxonne et plus particulièrement Marc américaine (Rand Corporation) et les difficultés Chopplet

Fellow IEA Nantes d'une définition. Il assignait aux années 90 en France l'émergence de telles organisations. Nous voudrions par ce dossier prolonger ce travail en nous intéressant plus particulièrement à une structure, le CESTA, créée une dizaine d'années auparavant au début des années 80 dans un contexte national et international particulier.

Le Centre d'Études des Systèmes et des Technologies Avancées (CESTA) fut peut-être le seul « think tank » français qui ait été en partie articulé sur la présidence de la République avec une mission de prévision et de prospective technologique 
mais également de mise en œuvre des grands programmes de coopérations internationales que furent « Technologie, Croissance, Emploi » à la suite du Sommet de Versailles des pays industrialisés en $1982^{2}$, et EURÊKA, en réponse à l'Initiative de Défense Stratégique (IDS) américaine en 1985. Cet EPIC ${ }^{3}$ (Établissement Public à caractère Industriel et Commercial) fut créé à la suite du Colloque National de la Recherche et de la Technologie ${ }^{4}$ de 1982, dans un contexte de victoire de la gauche, de mise en place des « programmes mobilisateurs » dans différents champs technologiques et de votes des premières lois de décentralisation. Il s'agissait alors de mettre en œuvre « une nouvelle alliance entre le monde de la recherche et les forces vives de la Nation ${ }^{5}$ ", ou encore, comme l'indiquait le président de la République dans son discours inaugural, de relever "le défi de la crise » (ce sont les premiers mots de son intervention) en rendant « au développement de la recherche et de la technologie son rôle prioritaire ${ }^{6} »$. Après les grands programmes sur 1'atome, l'espace et l'aéronautique des années 60, il s'agissait « d'imaginer »d'autres développements et d'associer « sur des grands objectifs nationaux, économiques, sociaux, culturels les efforts de l'appareil de recherche public et privé et de ceux de l'industrie et donner à ces efforts, sous l'impulsion de l'État, le dynamisme des grandes aventures humaines ${ }^{7} »$.

Lucien Sfez soulignait dans Technique et Idéologie que « ni les images, ni la fiction de la technique ne peuvent avoir d'action directe, et qu'il faut qu'elles soient relayées par les grands corps d'État pour s'imposer ${ }^{8}$ ». Ce point de vue de Lucien Sfez est confirmé par l'histoire du CESTA.
Jacques Attali, conseiller du président de la République, énarque et polytechnicien, joua un rôle déterminant dans sa création et dans les missions qui lui furent confiées (voir article de Brigitte Chamak dans ce dossier). Mais en même temps le CESTA de 1982 à 1986 tenta précisément de créer les conditions pour contourner le management des grands corps au profit d'une nouvelle organisation non plus pyramidale mais matricielle des interactions entre science, technologie et société en s'appuyant sur la prospective technologique comme instrument de désincarcération des logiques techniciennes. En ce sens, le CESTA fut un organisme atypique dans le paysage français. Son installation dans les anciens locaux de l'École polytechnique sur la Montagne SainteGeneviève, au cœur de Paris et à proximité immédiate du ministère, nouvellement créé, de la Recherche et de la Technologie ${ }^{9}$ qui ambitionnait de devenir l'équivalent du MITI japonais, est hautement symbolique. Ce positionnement proche du politique, comme la nature même de ses activités et de son personnel issu dans une large mesure du secteur des sciences humaines et sociales, en font un marqueur remarquable sur les attendus du politique et les stratégies de mobilisation des différents acteurs de la recherche, de l'industrie et des territoires en lien avec les lois de décentralisation de 1982. Sa dissolution même, à la suite du décès de son directeur général, Yves Stourdzé (1947-1986), est significative des jeux de pouvoir et des forces en présence pour le maintien d'un statu quo considérant que le développement des hautes technologies ne pouvait être conduit que par les très grands groupes industriels et leur management habituel. Un coup de force ou un coup de bluff ${ }^{10}$ pour lier le social, le biologique et le subjectif et permettre l'émergence de nouvelles 
communautés humaines ${ }^{11}$.

Ce dossier est en lien avec le colloque « Innovation et Prospective ; Yves Stourdzé explorateur et éclaireur des mondes à venir » qui s'est tenu les 18 et 19 novembre 2015 au ministère de l'Éducation nationale, de l'Enseignement Supérieur et de la Recherche en partenariat avec l'IHEST (Institut des Hautes Études Science et Technologie), les Universités Paris VIII et Bordeaux III et la MSH Paris-Nord. Il n'a pas pour objectif d'être exhaustif sur les activités et les travaux du CESTA. Il ne s'agit pas non plus d'établir un bilan de son action ou de dresser un portrait complet de son Directeur, Yves Stourdzé, qui y joua un rôle charismatique fort, aussi bien en interne qu'à travers les réseaux de coopération plurisectoriels, pluridisciplinaires et multipolaires qui furent créés. Il se propose d'une part de resituer ce «moment CESTA» dans son contexte et dans sa singularité et d'autre part de donner quelques clés pour en appréhender et comprendre la dynamique. Une manière aussi d'interroger les politiques publiques d'aujourd'hui dans le domaine de l'innovation et des coopérations internationales.

Brigitte Chamak (Université Paris 5) revient sur les origines et l'histoire du CESTA. Elle dégage d'une part les filiations avec le « Groupe des dix » qui se réunissait dans les années 1970 et d'autre part l'originalité et l'ampleur des travaux du CESTA. Elle montre ainsi l'inscription des orientations dans des courants de pensée visant à créer des conditions d'utilisation des connaissances scientifiques et des développement technologiques en vue de construire une société plus égalitaire, en même temps qu'elle souligne la spécificité de la démarche stratégique du CESTA sous la direction d'Yves Stourdzé et son implication notamment dans la mise en œuvre de coopérations scientifiques, technologiques et industrielles nouvelles au plan international. Elle fait enfin une place au rôle de la structure dans l'émergence des sciences cognitives en France.

Sous le titre « Le CESTA, une autre conception de l'innovation et de la prospective », je propose dans ce dossier une mise en perspective de la démarche proposée par Yves Stourdzé, et mise en œuvre au sein du CESTA, avec les travaux de prospective initiés en France depuis la fin des années 50 marqués par la création du Centre d'études prospective de Gaston Berger et la revue Futurible notamment. L'apport de l'ouvrage publié en 1978, Les Ruines du futur par Yves Stourdzé, marque un tournant. Il livre une lecture en rupture totale avec les travaux classiques de prospective et propose une vision de l'avenir entre bouleversements radicaux, innovations scientifiques et technologiques, irruption de nouvelles communautés à l'échelle mondiale dans une vision à la fois poétique, prophétique et politique qui va nourrir une nouvelle conception des rapports entre science, technologie et société et contribuer à repenser les contours des collaborations internationales.

Le CESTA fut une tentative de mise en œuvre d'une organisation matricielle répondant aux objectifs d'ouverture, de concertation et d'élaboration en réseaux de projets à géométries variables. Franck Cormerais (Université Bordeaux Montaigne) s'attache dans son article à revisiter la question des organisations par une relecture de l'ouvrage d'Yves Stourdzé 
Organisation, anti-organisation (1973, nouvelle édition 2015). Face à l'auto-transcendance actuelle des marchés et à la mystification d'une croissance illimitée, il souligne les enjeux d'une conception nouvelle de l'organisation ne reposant plus sur le sacrifice des libertés. À travers l'organisation il s'agit de penser autrement l'innovation et la prospective.

Par un exemple concret, celui des Nouvelles Technologies à l'Hôpital, Jean-Pierre Thierry (Médecin et Consultant) illustre les travaux engagés par le CESTA dans ce domaine. Il montre l'intérêt qu'ils auraient dans le contexte actuel, notamment à l'orée d'une transformation radicale de la recherche médicale avec l'apport des Big data. L'introduction de systèmes d'aide à la décision médicale et les investissements lourds consentis par plusieurs pays dans une période économique difficile en matière d'infrastructures informatiques, lui rappellent la période des projets Eurêka des années 80. Jugés indispensables pour relancer la recherche médicale et notamment la lutte contre le cancer, ils nécessitent plus que jamais de pouvoir bénéficier d'une vision prospective et donc de lieux de réflexion et d'animation facilitant l'émergence de nouveaux modèles nécessitant une modification des frontières entre disciplines et, sans doute, ajoute-t-il, la modernisation de certaines citadelles.

Enfin, Marc Lévy (Directeur de la prospective au GRET) rappelle le contexte des relations internationales au début des années 80 et le caractère pionnier d'une autre vision du monde qui tendait à s'imposer au GRET comme au CESTA. Il souligne en particulier la lenteur des évolutions du dispositif de coopération étalées sur plusieurs années (1998/2004/2008) qui a débouché finalement sur la création d'une « direction de la mondialisation et des partenariats » près de 30 ans après ces travaux pionniers.

À travers cette expérience particulière, ce « moment CESTA », à la fois se réalise concrètement l'articulation étroite entre technologie, communication et pouvoir et se révèle dans les rapports entretenus au sein de l'appareil d'État et dans les décisions de suppression de la structure, le potentiel dérangeant pour les pouvoirs de cette conjonction. En se positionnant moins comme aide à la décision que comme partie prenante et opérateur, le CESTA, franchissait une ligne rouge et passait d'une structure de prospective et de mission initiant des projets à une structure de pouvoir mettant en œuvre une stratégie multidimensionnelle (technologique, territoriale, internationale) d'organisation d'un nouvel espace-temps imaginatif faisant fi des contraintes pesant habituellement sur l'innovation et de développement. Face aux pesanteurs actuelles, au climat dépressif où nous guettent catastrophes économiques, catastrophes écologiques et catastrophes terroristes, une bouffée d'optimisme, de liberté, d'inventivité...

C'est peut-être à ça que doivent servir les think tanks? 


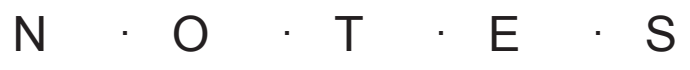

1. Think tanks, experts et pouvoirs, Quaderni n 70 , Automne 2008.

2. Technologie, Croissance, Emploi, Rapport aux sept chefs d'État et de Gouvernement et aux représentants des Communautés européennes, La Documentation française, janvier 1983.

3. Placé sous tutelle du ministère de la Recherche et de 1'Industrie, le CESTA devient EPIC par décret 83-126, le 22 février 1983.

4. Le colloque national Recherche et Technologie s'est tenu à Paris du 13 au 16 janvier 1982. Il avait été précédé de l'organisation de trente et une Assises Régionales. Le ministre de la Recherche et de la Technologie (Jean-Pierre Chevènement), tout comme le Premier ministre (Pierre Mauroy) annoncent dans leurs interventions de clôture la création du CESTA et d'un Institut de Prospective et de Prévision qui sera partie intégrante du CESTA. Actes du Colloque national Recherche et Technologie, La Documentation française, 1982, pp. 203, 207, 213.

5. Ibid. Intervention de François Gros, Conseiller auprès du Premier ministre et président du Comité d'organisation du colloque, p. 65.

6. Ibid. Discours de François Mitterrand le 13 janvier 1982, p. 67 : "Pour sortir de la crise, la recherche constitue l'une des clés essentielles, peut-être la clé du renouveau. Seul un gigantesque effort de recherche permettra à la France de prendre place parmi les quelques rares Nations capables de maîtriser leurs technologies et en définitive de conserver leur indépendance. Commençons par un acte de volonté, n'acceptons comme inéluctables ni les rapports économiques de domination, ni la division internationale du travail telle qu'elle est aujourd' hui». Discours de Pierre Mauroy : «La recherche et la technologie peuvent donc être la matrice qui nous permettra d'élaborer cette civilisation contemporaine, cette civilisation-urbaine et industrielle que le président de la République évoquait le 31 décembre dernier $»$, p. 211.

7. Ibid. p. 72.

8. L. Sfez, Technique et Idéologie, un enjeu de pouvoir, Éd du Seuil, 2002, p. 225.

9. Ministère désigné par François Mitterrand dans le Colloque national comme « avocat de l'avenir ». Opus cité, p. 68 et p. 211.

10. Comme le soulignait Lucien Sfez, Opus cité, p. 251. 11. Y. Stourdzé, «Le statut de l'opérateur humain dans les systèmes de communication » in Pour une poignée d'électrons, Pouvoir et communication, Fayard, 1987, Sens\&Tonka, 2016. Voir p. 290 notamment. Ces « nouveaux groupes humains » ressemblent à s'y méprendre à ceux des réseaux sociaux d'aujourd'hui. 
